\title{
L'holographie numérique pour la mesure 3D en mécanique des fluides
}

Des mesures 3D en mécanique des fluides sont requises pour recaler les modèles de turbulence, ou encore améliorer la compréhension d'écoulements complexes intervenant dans divers secteurs applicatifs : en géophysique (turbulence atmosphérique, transport de sédiments), dans le secteur médical (étude d'écoulements dans des microcavités), dans l'industrie automobile (étude de spray) ou encore dans l'industrie chimique (écoulements multiphasiques réactifs). Dans ce contexte, I'holographie numérique de micro-particules qui permet d'estimer la taille, la position et la vitesse d'objets répartis dans un volume apparaît comme une technique de mesure simple, précise et complémentaire aux autres techniques de mesures 3D.

Delphine CHAREYRON

Laboratoire de Physique - UMR CNRS 5672

École Normale Supérieure de Lyon,

Université de Lyon

delphine.chareyron@ens-lyon.fr

$\gg$ Corinne FOURNIER

Laboratoire Hubert Curien - UMR CNRS 5516

Université Jean Monnet,

Télécom Saint-Etienne

corinne.fournier@telecom-st-etienne.fr

$\gg$ Jean-Louis MARIÉ

Laboratoire de Mécanique des Fluides et

d'Acoustique (LMFA) - UMR CNRS 5509

École Centrale de Lyon/Université Lyon 1/INSA

jean-louis.marie@ec-Iyon.fr

\section{L'holographie numérique} pour la mécanique des fluides

\section{De l'holographie optique \\ à l'holographie numérique}

L'holographie est une méthode optique reconnue permettant d'enregistrer le «relief » d'un objet sur une plaque plane photosensible et de reconstruire cet objet en 3D. Elle utilise la cohérence de la lumière pour enregistrer la phase de I'onde lumineuse diffusée ou transmise par l'objet. L'holographie est appliquée depuis sa découverte à l'étude expérimentale d'écoulements fluides, pour mesurer la taille, la position ou encore la vitesse de particules présentes dans un écoulement. Cependant, malgré le potentiel important du procédé, I'holographie est longtemps restée une tech- nique de laboratoire. Cela est dû d'une part à la lourdeur associée au développement des plaques holographiques et d'autre part à la complexité du montage d'analyse.

Depuis une vingtaine d'années, les progrès technologiques en matière de caméras numériques, ont ouvert à l'holographie de nouvelles perspectives [1]. Avec des définitions accrues et des dynamiques plus importantes, l'acquisition d'hologrammes directement sur matrice CCD est aujourd'hui possible. Bien que la résolution et la taille des $C C D$ restent inférieures à celles des plaques holographiques, I'approche numérique permet des cadences d'acquisition élevées et rend la technique accessible aux nonopticiens grâce à une acquisition et une reconstruction d'image simplifiée.

Les traitements des images-hologrammes ont également beaucoup évolué ces dernières années, menant à une amélioration de la précision de la reconstruction $3 \mathrm{D}$ et à une augmentation de la largeur du champ d'étude accessible.

Une technique de suivi 3D et de mesure de taille en mécanique des fluides

Cette nouvelle holographie, dite numérique, est à la base de nouvelles techniques de diagnostic tridimensionnel utilisables dans le domaine expérimental. Les enjeux sont particulièrement importants dans le domaine de l'analyse d'écoulements.

Des outils de diagnostic optique (vitesse et/ou taille des particules portées par
I'écoulement) ont été proposés au cours de ces vingt dernières années : la vélocimétrie laser Doppler (LDV, Laser Doppler Velocimetry), I'anémométrie phase Doppler (PDA, Phase Doppler Anemometry), la vélocimétrie par image de particules (PIV, Particle Image Velocimetry), l'imagerie interférométrique par défaut de mise au point (ILIDS, Interferometric Laser Imaging for Droplet Sizing), la tomographie...

Ces instrumentations donnent accès à des informations en un point ou une partie de l'espace, on parle alors d'un point de vue Eulérien. Le point de vue Eulérien est plus facile à réaliser en laboratoire, mais ne permet pas de comprendre complètement les phénomènes physiques mis en jeu. En effet lorsque l'on s'intéresse par exemple à la dispersion de particules dans l'atmosphère ou aux problèmes de mélange, il paraît plus naturel de pouvoir suivre chaque particule le long de sa trajectoire, le point de vue Lagrangien devient alors plus naturel.

De fait, le volume de mesure de ces instrumentations est soit tout petit (environ $1 \mathrm{~mm}^{3}$ pour la LDV et la PDA), soit restreint à une coupe $2 \mathrm{D}$ de l'écoulement (PIV, ILIDS, tomographie). Il est en effet indispensable de disposer de mesures 3D pour progresser dans les domaines cités plus haut. En particulier la validation des simulations numériques d'écoulements turbulents manque d'informations 3D. Récemment, on a vu se développer différentes instrumentations Lagrangiennes $3 \mathrm{D}$ comme des techniques d'imagerie 
stéréo utilisant plusieurs caméras rapides pour le suivi 3D des particules dans un volume (ou 3D PTV - 3D Particle Tracking Velocimetry).

D'autres techniques de vélocimétrie basées sur l'effet Doppler (acoustique ou opti que ou eLDV, extended Laser Doppler Velocimetry, avec un volume de mesure 10 fois supérieur à celui de la LDV) donnent directement accès à la vitesse des particules le long de leurs trajectoires, sans informations sur la position.

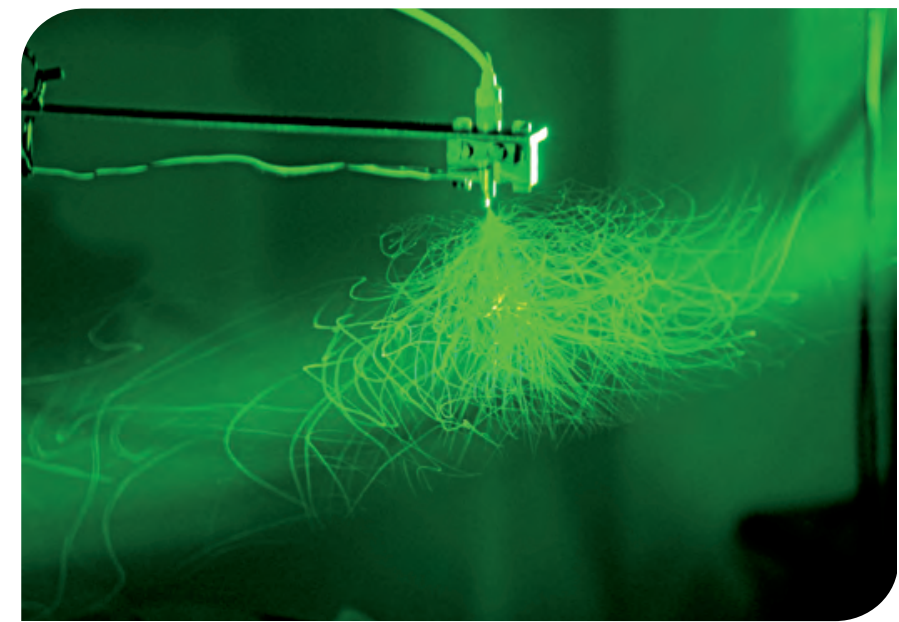

Figure 1. Dispersion d'un jet de gouttelettes d'eau dans un écoulement turbulent (pause longue).

holographie numérique est incontestablement une technique complémentaire, facile à mettre en œuvre, peu coûteuse et qui apporte une information supplémentaire (par rapport aux techniques de vélocimétrie) sur la taille des objets étudiés. Nous présentons sur la figure 1 des résultats obtenus à l'aide d'un montage d'holographie en ligne rapide pour étudier la dispersion d'un jet de gouttelettes d'eau (60 microns) dans un écoulement turbulent. La reconstruction d'une centaine de trajectoires est présentée sur la figure 2. Dans cette application, une très grande précision sur la mesure du diamètre des gouttelettes était requise. Les performances de ce montage sont illustrées sur la figure 3, où une seule trajectoire est représentée chronologiquement du cyan au bleu avec, en dessous, la mesure de son dia- mètre au cours du temps. La précision de la mesure atteint ici $2 \%$ de la taille des objets étudiés, ce qui est prometteur pour des applications où l'on s'intéresse à la variation de taille des objets au cours du temps (évaporation, dissolution, coalescence...).

Dans ce contexte, I'holographie numérique est une technique complémentaire permettant un positionnement 3D des objets tout en procurant une estimation précise de leur taille.

\section{Comment acquérir des hologrammes numériques?}

Différents montages holographiques existent : montage à deux bras, avec ou sans inclinaison de l'onde de référence.
Des montages plus complexes, exploitant la couleur, ont été dernièrement proposés (voir ci-après l'article signé par Jean-Michel Desse et Pascal Picart). Nous nous contentons ici de décrire le montage de Gabor (holographie en ligne) simple à mettre en œuvre et donc accessible aux non opticiens.

\section{Le montage en ligne}

Notons que I'holographie en ligne de microparticules a été I'une des premières applications métrologiques de l'holographie optique, car les objets (microparticules) créent des hologrammes interprétables à l'œil nu. Ce montage est également adapté à I'analyse de microparticules réparties dans un volume car il ne génère pas de trop hautes fréquences spatiales qui seraient mal échantillonnées par les capteurs numériques. Il présente l'énorme avantage d'être peu sensible aux vibrations car l'onde de référence et l'onde objet sont identiques, et il est donc utilisable en milieu industriel.

L'éclairement du capteur étant direct, l'énergie nécessaire à l'enregistrement d'un hologramme est faible ce qui permet des durées d'acquisition courtes et par conséquent l'analyse de phénomènes rapides. Le matériel mis en œuvre est simple et peu onéreux : diode laser, lentilles, caméra numérique.

$\gg$

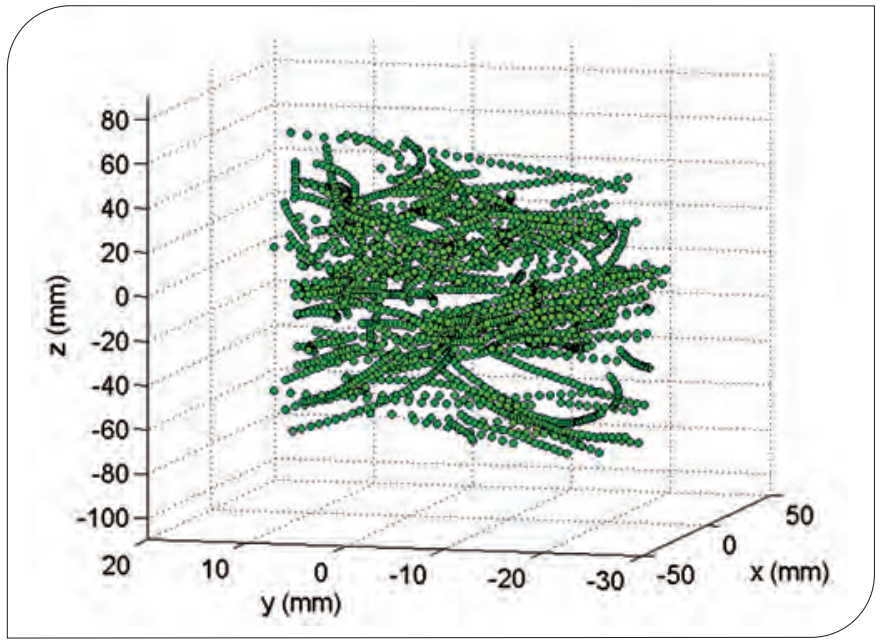

Figure 2. Reconstruction d'une centaine de trajectoires de gouttelettes d'eau (60 microns).

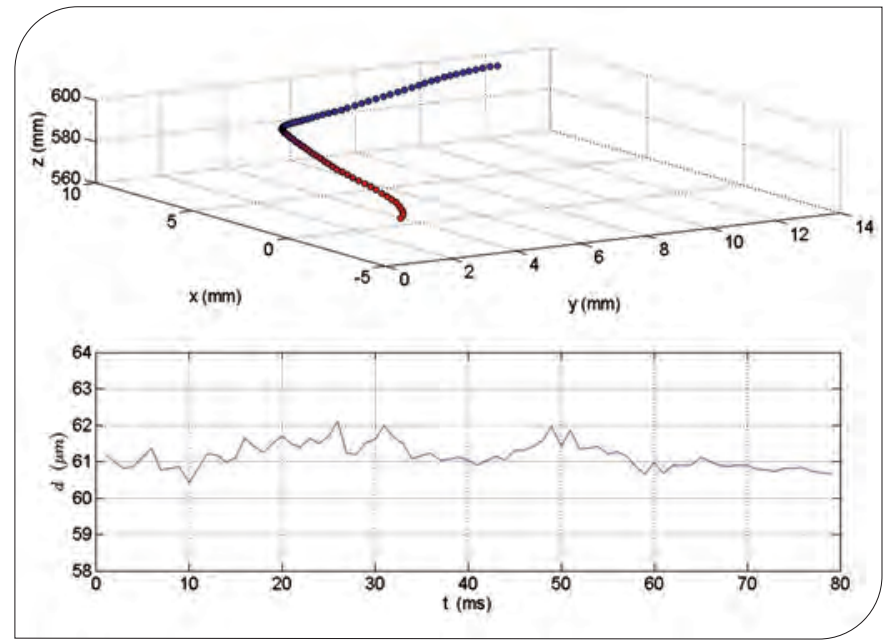

Figure 3. Une trajectoire de gouttelette d'eau chronologiquement du cyan au bleu, et la mesure de son diamètre au cours du temps. 


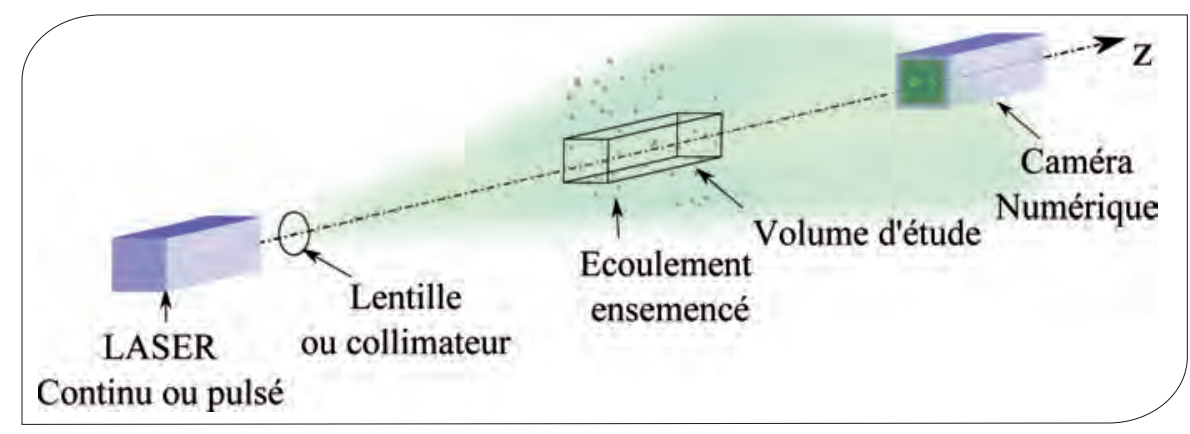

Figure 4. Montage d'enregistrement d'un hologramme numérique en ligne.

\section{Comment enregistrer un hologramme ?}

L'étape d'enregistrement consiste à éclairer l'écoulement ensemencé à l'aide d'un faisceau laser élargi et filtré (figure 4). Uncapteur numérique est positionné perpendiculairement au faisceau et enregistre les interférences entre la lumière diffractée par les particules (onde objet) et le faisceau divergent (onde de référence). Ces figures d'interférences sont des anneaux concentriques (figure 5). L'information de positionnement des particules est enregistrée dans la modulation fréquentielle de ces anneaux, alors que l'information de taille est enregistrée dans la fonction de contraste de ces anneaux. L'utilisation d'une caméra numérique permet de régler en direct la puissance du faisceau de référence et d'enregistrer des hologrammes successifs mono-exposés. Ceci permet de suivre l'évolution d'un nuage de particules. Le champ peut être transversalement ajusté par l'ajout d'un objectif devant le capteur ou par ajustement de la divergence de l'onde. Dans ce cas, une étape de calibrage doit être réalisée en translatant le long de l'axe optique une mire de microscope.

\section{Quel matériel utiliser?}

Le matériel à utiliser est identique à celui de la vélocimétrie par image de particules. Cependant certaines caractéristiques doivent être particulièrement prises en considération si l'on veut obtenir la meilleure précision possible.

\section{- Caméra}

Les figures de diffraction étant non localisées, la caméra doit avoir la plus grande
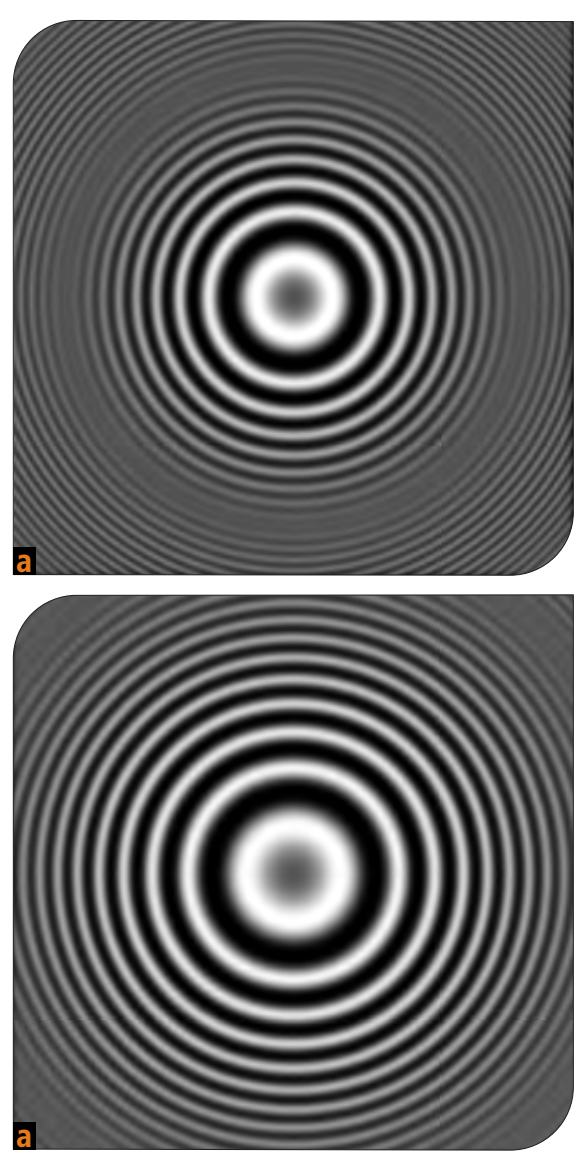

Figure 5. Exemples d'hologrammes simulés d'une particule située à : (a) $200 \mathrm{~mm}$, (b) $300 \mathrm{~mm}$ du capteur.

définition possible pour pouvoir enregistrer un maximum d'information. Ac tuellement on trouve sur le marché des caméras à $4000 \times 4000$ pixels. Cependant les fréquences d'acquisition de ces caméras sont faibles. On utilisera une définition minimum de $1024 \times 1024$ pixels (pour une telle définition la cadence des caméras rapides peut atteindre $10000 \mathrm{~Hz}$ ). Toujours dans le but d'enregistrer un maximum d'information, la dynamique de la caméra doit égale- ment être la plus grande possible. La taille du pixel n'est pas un paramètre fondamental mais elle influera sur la précision au même titre que la distance d'enregistrement.

\section{- Laser}

Contrairement à la vélocimétrie par image de particules, ce n'est pas la puissance du laser qui nous intéresse en holographie mais plutôt sa cohérence. Un laser de quelques centaines de milliwatt est suffisant. L'éclairement du capteur étant direct, il est indispensable d'ajouter des filtres de densité ou des polariseurs dans le montage pour ajuster la puissance du faisceau laser si celle-ci n'est pas réglable.

Un laser pulsé peut être utilisé pour éviter un effet de filé image (dû à une durée d'acquisition trop longue) ou réduire la durée d'exposition.

\section{Comment reconstruire}

les positions 3D et estimer les tailles des particules à partir des hologrammes 2D ?

\section{Restitution et reconstruction 3D}

La restitution d'un hologramme en ligne enregistré sur plaque photographique se fait par ré-éclairement de celui-ci par un faisceau identique à celui de l'enregistrement. Les figures d'interférences, surl'hologramme, se comportent comme des lentilles de Fresnel focalisant le faisceau en différents points correspondant aux positions des particules. En holographie numérique cette étape est faite par simulation numérique de la diffraction de l'hologramme éclairé par une onde plane (figure 6). Le calcul de l'intensité restituée en différents plans de l'espace permet de visualiser l'image du nuage de particules holographiées.

La reconstruction consiste à analyser les différents plans restitués. La position de chaque objet est estimée et le nuage de particules peut être reconstruit. Différents algorithmes ont été proposés ces dernières années pour extraire au mieux les positions et les diamètres des particules dans l'espace. L'information de positionnement en z étant enregistrée 


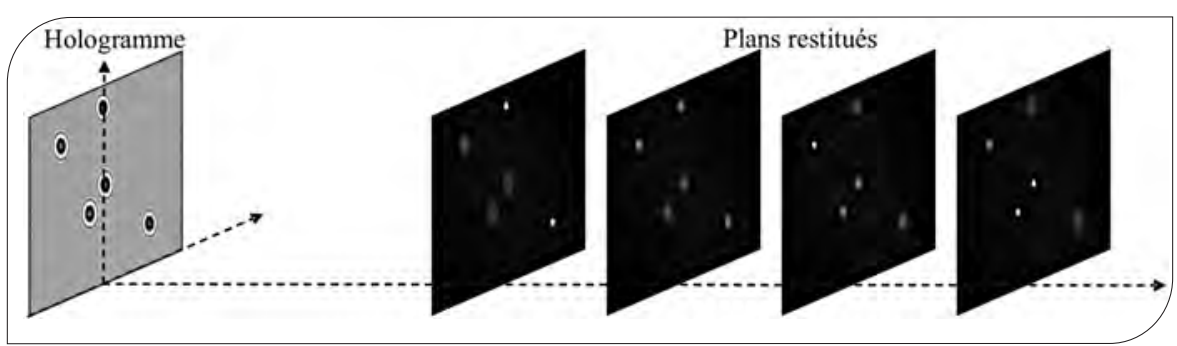

Figure 6. Illustration de la restitution numérique d'un hologramme.

dans la modulation des anneaux d'interférence, elle est intrinsèquement moins précise (de l'ordre de 50 fois moins) que l'information de positionnement transversal (en $x$ ou en $y$ ).

\section{Nouvelle approche de type «problèmes inverses »}

Une quantité importante d'information est contenue dans l'hologramme: forme, taille, orientation et position 3D des objets éclairés. L'extraction de cette information directement dans I'hologramme sans passer par les étapes de restitution puis de reconstruction est possible et réduit les artefacts de reconstruction. Nous avons proposé récemment [2] un traitement de type " problèmes inverses " permettant d'extraire l'information tridimensionnelle de I'hologramme de manière optimale.

Considérons un nuage de particules sphériques de positions et de diamètres connus, le problème direct consistant à simuler l'hologramme à partir de ces paramètres physiques est facile à résoudre. En effet la théorie de l'optique ondulatoire fournit un modèle paramétrique (dépendant des positions et des tailles des objets holographiés) de formation de l'hologramme (théorème d'Huyghens Fresnel). Le problème inverse, lui, con siste à retrouver les paramètres physiques à partir de l'hologramme enregistré : c'est le problème d'analyse d'un hologramme. Nous le résolvons en cherchant le maximum de vraisemblance entre un modèle et un hologramme. Le modèle de formation d'hologramme pouvant être (en première approximation) considéré comme additif, la recherche du meilleur modèle peut être réalisée itérativement particule par particule. Le modèle ajusté aux données est alors la figure de diffraction d'une particule. Après optimisation, chaque motif peut être soustrait de l'image (cleaning). Cette dernière étape permet d'augmenter le rapport signal sur bruit des particules en bord de champ ou hors champ. Leur détection devient alors possible (figure 7) tout en limitant le risque de détection multiple d'une même particule. Cet algorithme est disponible à l'adresse de la référence [3] sous forme d'une Toolbox Matlab.

Notons que cette approche (parfois nommée de type "compressed sensing ») peut être étendue à des objets quelconques [4].

\section{Quelques exemples}

L'algorithme ci-dessus a été appliqué à des hologrammes d'un jet monodisperse de gouttelettes d'eau en chute libre. Le montage est illustré figure 4 et les résultats figure 7. La taille du champ (classiquement limitée à la taille du capteur) a pu être multipliée par 4 grâce à l'utilisation d'une méthode de reconstruction de type "problèmes inverses » sans perte de précision. Les diamètres des gouttelettes ont pu être estimés avec un écart type de l'ordre du micron.

\section{Suivi de gouttelettes évaporantes}

Dans cette expérience [5], des gouttelettes de fréon (diamètre 200 microns, température d'ébullition : $3,8^{\circ} \mathrm{C}$ ) sont injectées autour de $0^{\circ} \mathrm{C}$ dans un écoulement turbulent gazeux à $20^{\circ} \mathrm{C}$ (figure 8). L'holographie numérique en ligne a permis de visualiser un sillage derrière chaque gouttelette. La visualisation de ce sillage est particulièrement prometteuse pour la mécanique des fluides. $\gg$

\section{Exemple de reconstruction hors champ}

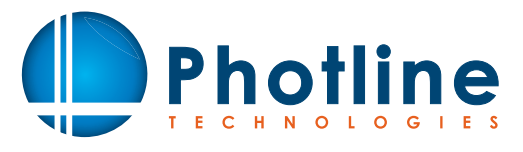

Delivering Modulation Solutions
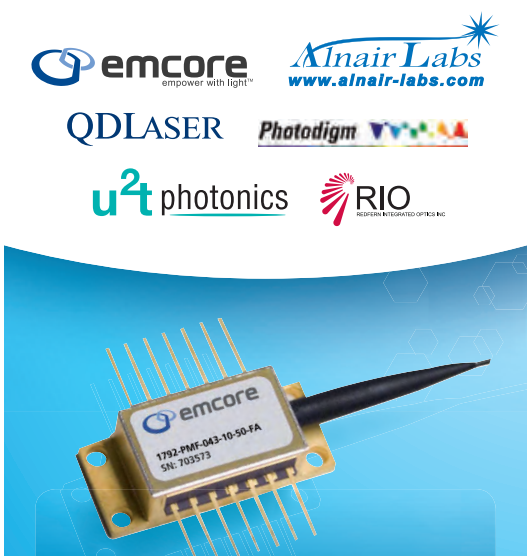

: Lesers

- Accordables bande C, bande L

- DFB faible RIN jusqu'à $100 \mathrm{~mW}$

- Spectre étroit : jusqu'à $3 \mathrm{kHz}$

- $780 \mathrm{~nm}, 850 \mathrm{~nm}, 1053 \mathrm{~nm}$,

- $1064 \mathrm{~nm}, 1300 \mathrm{~nm}, 1550 \mathrm{~nm}$

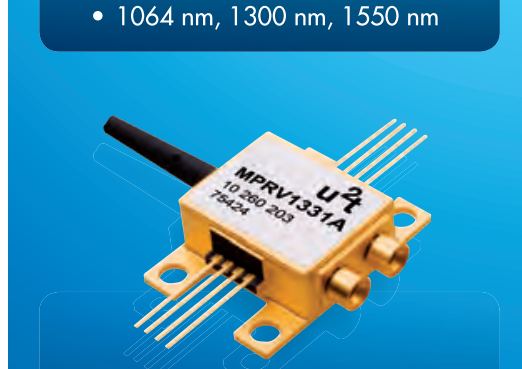

a: Photodétecteurs

- $10 \mathrm{GHz}, 20 \mathrm{GHz}, 40 \mathrm{GHz}$, $70 \mathrm{GHz}, 100 \mathrm{GHz}$

- Numériques, analogiques

- Equilibrés pour détection cohérente

- Récepteurs intégrés DPSK, DQPSK

5

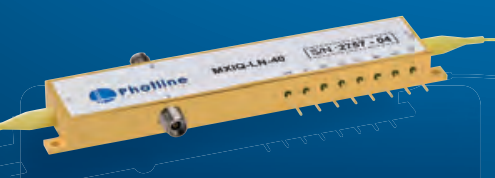

$\because:$ Modulateurs $\mathrm{LiNbO}_{3}$

- Intensité et phase

- Jusqu'à $40 \mathrm{~Gb} / \mathrm{s}-40 \mathrm{GHz}$

- $1550 \mathrm{~nm}, 1310 \mathrm{~nm}$

- $800 \mathrm{~nm}, 1000 \mathrm{~nm}, 2 \mu \mathrm{m}$

contact@photline.com

Tél. : 0381853180

www.photline.com 


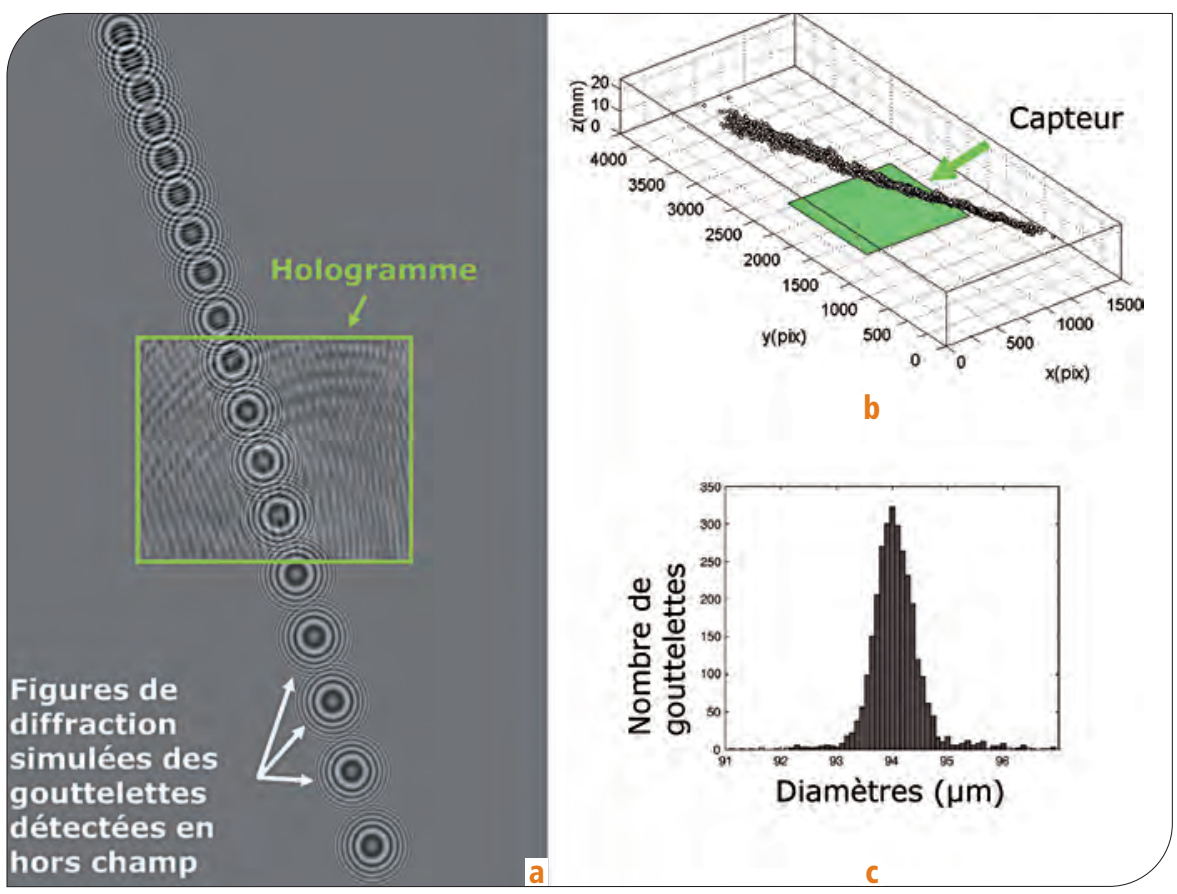

Figure 7. Illustration de la détection hors champ sur un jet de gouttelettes monodisperses en chute libre : (a) Superposition d'un hologramme expérimental et de l'hologramme simulé en hors champ, (b) Reconstruction 3D de 200 hologrammes, (c) Distribution des diamètres des gouttelettes holographiées.

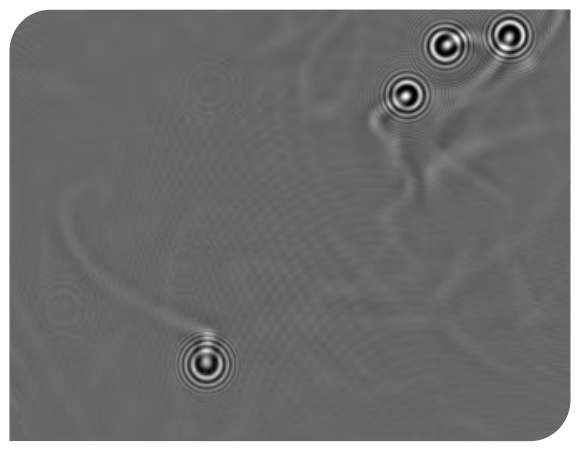

Figure 8. Exemple d'hologramme de gouttelettes de fréon (200 microns de diamètre) acquis à 3000 images/s.

En effet, il donne accès à la topologie du champ de concentration, et par suite au taux d'évaporation local. Ces informations sont de tout premier ordre lors qu'on s'intéresse, par exemple, à des problèmes de combustion. En visionnant le film de l'enregistrement, si l'on s'intéresse plus particulièrement à l'évolution du sillage par rapport aux trajectoires des gouttelettes, il est possible d'avoir une information (2D) sur la vitesse relative de l'écoulement autour de chaque goutte. Le sillage est comme un scalaire passif qui vient marquer l'écoulement porteur vu dans le référentiel de chaque goutte. Une mise au point numérique dans le plan de la goutte $n$ 'apporte pas d'information supplémentaire car l'image de ce sillage ne correspond pas à une figure d'interférence créée par un objet mais à un gradient d'indice derrière la goutte. Ainsi, un montage d'holographie avec un deuxième bras à $90^{\circ}$ permettrait $d^{\prime}$ avoir une information 3D de la vitesse relative de l'écoulement par rapport aux particules.

\section{Discussion - Conclusion}

L'holographie reste une des seules techniques de mesure en mécanique des fluides permettant d'estimer la taille, la position, la vitesse d'objets répartis dans un volume. Comme toute technique elle possède ses limites, fortement liées aux paramètres expérimentaux, dont nous donnons ici quelques ordres de grandeur :

- le nombre d'objets ne doit pas être trop important (environ moins d'une centaine de particules) ;

- pour des raisons de contraste la taille des objets ne doit pas être inférieure à environ $10 \mu \mathrm{m}$. Dans un montage à gran- dissement un, c'est-à-dire sans objectif, cette taille minimale peut être diminuée mais au détriment du champ d'étude par rapport au cas d'un grandissement supérieur à 1 ;

- la précision transversale (perpendiculairement à l'axe optique) est 50 fois plus élevée que la précision longitudinale (profondeur).

L'holographie numérique en ligne présente aussi de nombreux atouts : estimation de la taille, la position, la vitesse d'objets répartis dans un volume avec une grande précision, de l'ordre du micron pour l'estimation de taille, inférieure au micron pour l'estimation des coordonnées transversales et de l'ordre de la dizaine de micron pour l'estimation de la profondeur.

L'holographie numérique en ligne présente aussi l'énorme avantage d'être simple et rapide à mettre en œuvre (environ une demi-journée pour une personne inexpérimentée) et utilisable sur des objets solides ou liquides. Dans un contexte industriel ces avantages sont primordiaux. De plus, les dernières avancées technologiques dans le domaine des caméras rapides, avec des résolutions de plus de un million de pixels à une cadence de plus de 10000 images par seconde, permettent un suivi Lagrangien d'objets, avec l'accès aux positions et tailles des objets ainsi qu'à la topologie des champs de concentration ou de température.

\section{Références}

[1] T. M. Kreis, Handbook of holographic interferometry. Akademie Verlag, 1996.

[2] F. Soulez, L. Denis, E. Thiébaut, C. Fournier, et C. Goepfert, «Inverse problem approach in particle digital holography: out-of-field particle detection made possible ", Journal of the Optical Society of America A, vol. 24, no. 12, p. 3708-3716, 2007.

[3] http://labh-curien.univ-st-etienne.fr/wiki-reconstruction

[4] L. Denis, D. Lorenz, E. Thiébaut, C. Fournier, et $D$. Trede, "Inline hologram reconstruction with sparsity constraints \#, Optics Letters, vol. 34, no. 22, p. 3475-3477, 2009.

[5] D. Chareyron, J.L. Marié, J. Gire, C. Fournier, N. Grosjean, L. Denis, L. Méès, M. Lance, "Mesures Lagrangiennes de gouttes évaporantes dans une turbulence homogène isotrope par holographie numérique», $1^{\text {ere }}$ rencontre francophone d'holographie numérique appliquée à la métrologie des fluides (Holophi), 2010, Rouen.

[6] http://www.Imfa.ec-lyon.fr/ 